\title{
Topology on words
}

\author{
Cristian S. Calude ${ }^{\mathrm{a}}$, Helmut Jürgensen ${ }^{\mathrm{b}}$, Ludwig Staiger ${ }^{\mathrm{c}}$ \\ a The University of Auckland, New Zealand \\ ${ }^{\mathrm{b}}$ The University of Western Ontario, London, Canada \\ ${ }^{\mathrm{c}}$ Martin-Luther-Universität Halle-Wittenberg, Germany
}

\section{A R T I C L E I N F O}

\section{Keywords:}

Formal languages

Combinatorics on words

Topology on words

$\omega$-languages

Order-based topologies

\begin{abstract}
A B S T R A C T
We investigate properties of topologies on sets of finite and infinite words over a finite alphabet. The guiding example is the topology generated by the prefix relation on the set of finite words, considered as a partial order. This partial order extends naturally to the set of infinite words; hence it generates a topology on the union of the sets of finite and infinite words. We consider several partial orders which have similar properties and identify general principles according to which the transition from finite to infinite words is natural. We provide a uniform topological framework for the set of finite and infinite words to handle limits in a general fashion.
\end{abstract}

(c) 2009 Elsevier B.V. All rights reserved.

\section{Introduction and preliminary considerations}

We investigate properties of various topologies on sets of words over a finite alphabet. When $X$ is a finite alphabet, one considers the set $X^{*}$ of finite words over $X$, the set $X^{\omega}$ of (right-)infinite words over $X$ and the set $X^{\infty}=X^{*} \cup X^{\omega}$ of all words over $X$. On the set $X^{\infty}$ concatenation (in the usual sense) is a partial binary operation defined on $X^{*} \times X^{\infty}$.

Infinite words are commonly considered limits of sequences of finite words in the following sense. A finite word $u$ is said to be a prefix of a $w \in X^{\infty}$, written as $u \leq_{\mathrm{p}} w$, if there is a word $v \in X^{\infty}$ such that $w=u v$; when $u \neq w$, $u$ is a proper prefix of $w$, written as $u<_{\mathrm{p}} w$. Consider an infinite word $w$, and an infinite sequence $w_{1}<_{\mathrm{p}} w_{2}<_{\mathrm{p}} w_{3}<_{\mathrm{p}} \cdots<_{\mathrm{p}} w$. Then it is natural to consider $w$ as the $\operatorname{limit}_{\lim } \rightarrow \infty w_{n}$. This observation suggests the definition of a topology on $X^{*}$, the well-known prefix topology. It is a topology on $X^{*}$ only and not on $X^{\infty}$; thus, the convergence of a sequence of finite words to a limit in $X^{\omega}$ is not easily explained in topological terms. To address this issue, one would need to consider a topology defined by the partial order $\leq_{\mathrm{p}}$ on $X^{\infty}$ in which limits have the desired behaviour.

Given any partial order $\leq$ on a set $S$, it seems to be natural to consider the topology Top $_{\leq}$generated by the family of sets $\{w \mid w \in S, u \leq w\}_{u \in S}$ as open sets. In the case of $S=X^{*}$, several partial orders different from $\leq_{\mathrm{p}}$ have been studied, mainly in the context of defining classes of codes [31]. By comparing the resulting topologies and their implied concepts of limit, adherence, continuity and so on, we expect to uncover general properties of topologies on words.

Our present investigation was motivated by the work on independence in mathematical theories, [6] by Calude, Jürgensen and Zimand, in which it was shown that the set of independent true statements in a sufficiently rich mathematical theory is "topologically large". To avoid trivial exceptions this result needed to be proved to hold for any "reasonable" topology on $X^{*}$; indeed, if the result were restricted to just one topology, the prefix topology, for instance, one could argue that the statement is not so much about independence, but about the idiosyncrasies of that topology. This thought initiated a systematic investigation of "reasonable" topologies, mainly such topologies which are naturally associated with classes of codes. While the result in [6] is expressed with respect to a class of topologies vastly greater than the class of topologies on $X^{*}$ defined by partial orders, the problem of understanding, in detail, the properties of the latter type of topology remained open.

We attempt to close this gap in the following sense. Let $X$ and $Y$ be finite alphabets; let $\leq$ be a partial order defined "in the same way" on $X^{*}$ and on $Y^{*}$. We consider the induced topology Top $\leq$ on $X^{*}$, which we then extend to $X^{\infty}$. Suppose 
$\varphi: X^{*} \rightarrow Y^{*}$ is monotone with respect to $\leq$. How can the mapping $\varphi$ be extended, in a natural fashion, to a mapping $\bar{\varphi}: X^{\infty} \rightarrow Y^{\infty} ?$

In particular, we investigate which partial orders on $X^{*}$ yield reasonable extensions. It turns out that prefix-based partial

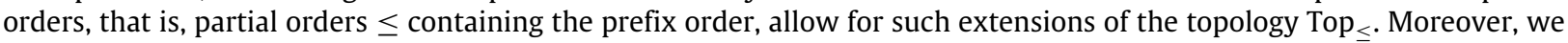
consider properties of the limits defined with respect to these topologies on $X^{*}$ and their extensions. Specifically, we explore to which extent topologies derived from such partial orders $\leq$ support a natural description of infinite words as limits of sequences of finite words thus allowing for the extension of $\leq$-monotone mappings as indicated above. An important issue is, how to present an infinite word $\xi \in X^{\omega}$ as a limit of sequences, of order type $\omega$, of finite words $\left(w_{j}\right)_{j \in \mathbb{N}}$ in such a way that $\xi$ is a limit point of $\left(w_{j}\right)_{j \in \mathbb{N}}$ if and only if $w_{0}<w_{1}<\cdots<w_{j}<\cdots<\xi$.

In the case of the prefix order $\leq_{\mathrm{p}}$, the concept of adherence plays a crucial rôle in extending continuous, that is, $\leq_{\mathrm{p}}$-monotone, mappings from $X^{*}$ to $X^{\omega}$. We apply the ideas leading to the definition of adherence to partial orders different from the prefix order. We then investigate the properties of the resulting generalized notion of adherence with respect to limits.

Several fundamentally different ways of equipping the set $X^{*}$ with a topology are proposed in the literature. Roughly, these can be classified as follows:

- Topologies arising from the comparison of words.

- Topologies arising from languages, that is, sets of words.

- Topologies arising from the multiplicative structure.

A similar classification can be made for topologies on $X^{\omega}$ and $X^{\infty}$. For $X^{\infty}$, topologies have not been studied much; however, to achieve a mathematically sound transition between $X^{*}$ and $X^{\omega}$, precisely such topologies are needed.

Our paper is structured as follows. In Section 2 we introduce notation and review some basic notions. In Sections 4 and 5 we briefly discuss topologies for the sets of finite and of infinite words as considered in the literature. General background regarding topologies and specifics relevant to topologies on words are introduced in Section 3. In Section 6 we consider extensions of partial orders on $X^{*}$ to $X^{\omega}$. Intuitively, the limits are related to reading from left to right, that is, according to the order type $\omega$; topologies derived from partial orders rely on this idea. In Section 7 we explore this intuition. Section 8 provides a discussion of special cases. In Section 9 we summarize the ideas and discuss the results.

A preliminary version of this paper was presented at the Joint Workshop Domains VIII and Computability Over Continuous Data Types, Novosibirsk, September 11-15, 2007 [5].

\section{Notation and basic notions}

We introduce the notation used and also review some basic notions.

By $\mathbb{N}$ we denote the set $\{0,1, \ldots\}$ of non-negative integers; $\mathbb{R}$ denotes the set of real numbers; let $\mathbb{R}_{+}$be the set of nonnegative real numbers.

For a set $S$, card $S$ is the cardinality of $S$, and $2^{S}$ is the set of all subsets of $S$. If $T$ is also a set then $S^{T}$ is the set of mappings of $T$ into $S$. The symbol $\omega$ denotes the smallest infinite ordinal number. As usual, $\omega$ is identified with the set $\mathbb{N}$. Thus $S^{\omega}$ is the set of all mappings of $\mathbb{N}$ into $S$, hence the set of all infinite sequences of elements of $S$. When considering singleton sets, we often omit the set brackets unless there is a risk of confusion.

An alphabet is a non-empty, finite set. The elements of an alphabet are referred to as symbols or letters. Unless specifically stated otherwise, every alphabet considered in this paper has at least two distinct elements.

Let $X$ be an alphabet. Then $X^{*}$ denotes the set of all (finite) words over $X$ including the empty word $\varepsilon$, and $X^{+}=X^{*} \backslash\{\varepsilon\}$. The set $X^{\omega}$ is the set of (right-)infinite words over $X$. Let $X^{\infty}=X^{*} \cup X^{\omega}$. With $\gamma \in\{*, \omega, \infty\}$ a $\gamma$-word is a word in $X^{\gamma}$. Similarly, a $\gamma$-language is subset of $X^{\gamma}$. When we do not specify $\gamma, \gamma=\infty$ is implied. For a word $w \in X^{\infty},|w|$ is its length.

On the set $X^{\infty}$ concatenation (in the usual sense) is a partial binary operation defined on $X^{*} \times X^{\infty}$. With concatenation as operation $X^{*}$ is a free monoid and $X^{+}$is a free semigroup; moreover, $X^{\infty}$ can be considered as a left act (also called a left operand $)^{1}$ resulting in a representation of the monoid $X^{*}$ as a monoid of (left) transformations of the set $X^{\infty}$.

We also consider the shuffle product w which is defined as follows: For $u \in X^{*}$ and $w \in X^{\infty}$,

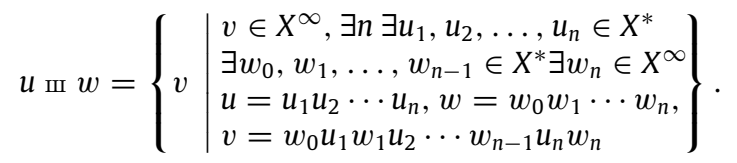

We consider binary relations $\varrho \subseteq X^{*} \times X^{\infty}$ and their restrictions to $X^{*} \times X^{*}$. Unless there is a risk of confusing the relations the latter is also just denoted by $\varrho$. Usually, such a relation is defined by some property of words, say $P$, and we write $\varrho_{P}$ to indicate this fact. When the restriction of $\varrho_{P}$ to $X^{*} \times X^{*}$ is a partial or strict order, we write $\leq_{P}$ or $<_{P}$, respectively.

The following relations play a special rôle in this paper, where $u \in X^{*}$ and $v \in X^{\infty}$ :

\footnotetext{
1 See [13] for basic definitions.
} 
- Prefix order: $u \leq_{\mathrm{p}} v$ if $v \in u X^{\infty}$.

- Infix order: $u \leq_{\mathrm{i}} v$ if, for some $w \in X^{*}, v \in w u X^{\infty}$.

- Embedding (or shuffle) order: $u \leq_{\mathrm{e}} v$ if, for some $w \in X^{\infty}, v \in u$ ш $w$.

For the next definitions we need a total ordering on the alphabet $X$ as afforded, for instance, by a bijective mapping $\alpha$ of $X$ onto the set $\{1,2, \ldots, q\}$ where $q=\operatorname{card} X$. Let $u=u_{1} u_{2} \cdots u_{n}$ and $v=v_{1} v_{2} \cdots$ with $u_{1}, u_{2}, \ldots, v_{1}, v_{2}, \ldots \in X$.

- Lexicographic order: If $u \not_{\mathrm{p}} v$ and $v \not_{\mathrm{p}} u$, let $i_{0}=\min \left\{i \mid u_{i} \neq v_{i}\right\}$. Then $u \leq_{\text {lex }} v$ if $u \leq_{\mathrm{p}} v$ or if $u \not \not_{\mathrm{p}} v, v \not \not_{\mathrm{p}} u$ and $\alpha\left(u_{i_{0}}\right)<\alpha\left(v_{i_{0}}\right)$.

- Quasi-lexicographic (or pseudo-lexicographic) order: $u \leq_{\mathrm{q}-\mathrm{lex}} v$ if $|u|<|v|$ or if $|u|=|v|$ and $u \leq_{\operatorname{lex}} v$.

If $\leq$ is any one of these relations, then $u<v$ if $u \leq v$ and $u \neq v$.

For a more comprehensive list of important binary relations, especially partial orders, on finite strings and their rôles in the definition of classes of languages or codes see [31,54,65].

Let $\leq$ be a partial order on $X^{*}$. The right extension of $\leq$ to $X^{*} \times X^{\infty}$ is defined as follows: For $u \in X^{*}$ and $v \in X^{\omega}$, $u \leq v$ if there is a word $w \in X^{*}$ such that $w \leq_{\mathrm{p}} v$ and $u \leq w$. For $v \in X^{\infty}$, the set

$$
\text { Pred }_{\leq} v=\left\{u \mid u \in X^{*}, u \leq v\right\}
$$

is the set of predecessors of $v$ with respect to $\leq$. The set

$$
\text { Succ }_{\leq} v=\left\{u \mid u \in X^{\infty}, v \leq u\right\}
$$

is the set of successors of $v$ with respect to $\leq$. In particular, Succ $\leq v=\emptyset$ for $v \in X^{\omega}$. For $L \subseteq X^{\infty}$, let

$$
\operatorname{Pred}_{\leq} L=\bigcup_{v \in L} \operatorname{Pred}_{\leq} v \text { and } \text { Succ }_{\leq} L=\bigcup_{v \in L} \operatorname{Succ}_{\leq} v .
$$

Specifically, we define Pref $=\operatorname{Pred}_{\leq \mathrm{p}}$ and Inf $=\operatorname{Pred}_{\leq_{\mathrm{i}}}$.

A *-language $L$ is said to be prefix-free (or a prefix code) if, for all $u, v \in L, u \leq_{\mathrm{p}} v$ implies $u=v$. Similarly, $L$ is infixfree (or an infix code) if, for all $u, v \in L, u \leq_{\mathrm{i}} v$ implies $u=v$. In general, for a binary relation $\varrho$, a language is $\varrho$-free (or $\varrho$-independent) if, for all $u, v \in L,(u, v) \in \varrho$ implies $u=v$. For further details concerning $\varrho$-freeness and codes see [31].

\section{General topologies}

We now present some basic background concerning topologies; we use [21,35] as general references. For topologies on partially ordered sets see also [3,39]

\subsection{Definitions}

A topology $\tau$ on a set $X$ is a pair $\tau=(\mathcal{X}, \mathcal{O})$ where $\mathcal{O} \subseteq 2^{X}$ is a family of subsets, called open sets, containing $\mathcal{X}$ itself and being closed under finite intersections and arbitrary unions. Alternatively, a topology on $\mathcal{X}$ can be defined by a closure operator $\mathrm{cl}: 2^{x} \rightarrow 2^{x}$ having the following properties:

$$
\begin{aligned}
M & \subseteq \operatorname{cl}(M) \\
\operatorname{cl}(M) & =\operatorname{cl}(\operatorname{cl}(M)) \\
\operatorname{cl}\left(M_{1} \cup M_{2}\right) & =\operatorname{cl}\left(M_{1}\right) \cup \operatorname{cl}\left(M_{2}\right) \text { and } \\
\operatorname{cl}(\emptyset) & =\emptyset
\end{aligned}
$$

A set $M$ satisfying $\operatorname{cl}(M)=M$ is said to be closed; the family of all complements of closed sets $\mathcal{O}=\{M \mid M \subseteq X \wedge \operatorname{cl}(X \backslash M)=$ $X \backslash M\}$ is closed under finite intersection and arbitrary union, hence a family of open sets.

A basis of a topology $\tau=(\mathcal{X}, \mathcal{O})$ is a family $\mathscr{B} \subseteq 2^{X}$ such that every $M \in \mathcal{O}$ is a union of sets in $\mathscr{B}$. A sub-basis of a topology $\tau=(\mathcal{X}, \mathcal{O})$ is a family $\mathscr{B}^{\prime} \subseteq 2^{X}$ such that the family $\left\{\bigcap_{j=1}^{n} M_{j} \mid n \in \mathbb{N} \wedge M_{j} \in \mathscr{B}^{\prime}\right.$ for $\left.1 \leq j \leq n\right\}$ is a basis of $\tau$. Every family $\mathscr{B}^{\prime} \subseteq 2^{X}$ when used as a sub-basis defines a topology on $\mathcal{X}$.

A point $x \in \bar{X}$ is an accumulation point of a set $M \subseteq X$ when $x \in \operatorname{cl}(M \backslash\{x\})$. This condition is equivalent to that of every open set $M^{\prime}$ which contains $x$ satisfying that $M^{\prime} \cap(M \backslash\{x\}) \neq \emptyset$. One can define the closure via accumulation points:

$$
\operatorname{cl}(M)=M \cup\{x \mid x \text { is an accumulation point of } M\} .
$$

For a topological space $(\mathcal{X}, \mathcal{O})$ and a subset $M \subseteq \mathcal{X}$ the pair $\left(M, \mathcal{O}_{M}\right)$ with $\mathcal{O}_{M}=\left\{M \cap M^{\prime} \mid M^{\prime} \in \mathcal{O}\right\}$ is the subspace topology on $M$ induced by $(\mathcal{X}, \mathcal{O})$. Here $\mathscr{B}_{M}=\left\{M \cap M^{\prime} \mid M^{\prime} \in \mathscr{B}\right\}$ is a basis for $\left(M, \mathcal{O}_{M}\right)$ if $\mathscr{B}$ is a basis for $(\mathcal{X}, \mathcal{O})$. 


\subsection{Sequences and limits}

A sequence in a space $X$ is an ordered family $\left(x_{j}\right)_{j \in \mathbb{N}}$ where $x_{j} \in \mathcal{X}$ but not necessarily $x_{i} \neq x_{j}$ for $i \neq j$, that is, such a sequence is an element of $\mathcal{X}^{\mathbb{N}}$. A point $x$ in a topological space $(\mathcal{X}, \mathcal{O})$ is called a limit point of the sequence $\left(x_{j}\right)_{j \in \mathbb{N}}$ if, for every open set $M \in \mathcal{O}$ containing $x$, there is $j_{0} \in \mathbb{N}$ such that $x_{j} \in M$ for all $j, j \geq j_{0}$. The set of all limit points of a sequence $\left(x_{j}\right)_{j \in \mathbb{N}}$ is denoted by $\lim x_{j}$. Observe that a sequence may have more than one limit point or no limit point at all.

In general topological spaces limit points of sequences are not sufficient to determine closed sets. In metric spaces the situation is different. Only the following holds true in general (see [21, Ch. I.6]).

Theorem 1. If a topological space $(\mathcal{X}, \mathcal{O})$ has a countable basis then for every $M \subseteq X$ its closure $\operatorname{cl}(M)$ is the set of all limit points of sequences $\left(x_{j}\right)_{j \in \mathbb{N}}$ where $x_{j} \in M$ for all $j \in \mathbb{N}$.

A cluster point of a sequence $\left(x_{j}\right)_{j \in \mathbb{N}}$ is a point $x$ such that for every open set $M^{\prime}$ containing $x$ there are infinitely many $j$ such that $x_{j} \in M^{\prime}$ (see [21]). Similarly, a point $x \in X$ is a cluster point of a set $M \subseteq X$ if, for every open set $M^{\prime}$ containing $x$, the intersection $M^{\prime} \cap M$ is infinite.

Remark 2. Every cluster point of $M$ is also an accumulation point of $M$. In spaces where every finite set is closed, every accumulation point is also a cluster point.

The difference in the definitions of accumulation and cluster points is useful in what follows, as most of the spaces considered in this paper have finite subsets which are not closed.

\subsection{Right topology}

In this last preliminary part we recall the concept of right (or ALEXANDROv) topology $\boldsymbol{\alpha}_{\leq}$on a set $\mathcal{X}$ partially ordered by some relation $\leq$. This topology is generated by the basis of right-open intervals $\mathbf{B}_{x}=\{y \mid y \in X \wedge x \leq y\}$. It has the following properties (see [21]).

Proposition 3. Let $(\mathcal{X}, \leq)$ be a partially ordered set, and let $\boldsymbol{\alpha}_{\leq}$be defined as $\left(\mathcal{X}, \mathcal{O}_{\leq}\right)$where $\mathcal{O}_{\leq}=\left\{\bigcup_{x \in M} \mathbf{B}_{x} \mid M \subseteq X\right\}$. Then the following hold true.

(1) $\mathbf{B}_{x}$ is the smallest open set containing $x$.

(2) An arbitrary intersection of open sets is again open.

(3) For every pair $x, y \in X$ there is an open set containing one of the points but not the other. In particular, if $y \notin x$ then $x \notin \mathbf{B}_{y}$.

(4) A point $x \in X$ is an isolated point, that is, the set $\{x\}$ is open, if and only if $x$ is a maximal element with respect to $\leq$ in $X$.

Note that, because of Property $3, \boldsymbol{\alpha}_{\leq}$is a $\mathrm{T}_{0}$ topology.

\section{Review of topologies for finite words}

Several fundamentally different ways of equipping the set $X^{*}$ with a topology are proposed in the literature, roughly classified as follows:

- Topologies arising from the comparison of words.

- Topologies arising from languages.

- Topologies arising from the multiplicative structure.

In most cases, the intended application of the topology requires that $X^{*}$ with the topology be a metric space.

Topologies related to $X^{*}$ arise also when one considers the space of formal power series $R\langle\langle X\rangle\rangle$ with a semiring $R$ as the coefficient domain and with the elements of $X$ as non-commuting variables (see [34], for example).

\subsection{Topologies from comparing words}

At least two methods have been proposed for comparing words and deriving topologies from them. One of the historical origins is the theory of codes, where the size and, implicitly, the improbability of an error are measured in terms of the difference between words. ${ }^{2}$ When only words of the same length are compared, as is the case in the theory of error correcting codes, the Hamming or the Lee metric, depending on the physical context, is commonly used. The Hamming metric just counts the number of positions in which two words of the same length differ; the Lee metric assumes a cyclic structure on the alphabet $X$ and reflects the sum of the cyclic differences of two words of the same length. Neither of these metrics seems to lead to a meaningful topology on the whole of $X^{*}$.

Also originating with the theory of codes is the Levenshtein distance [37] between words of arbitrary length; sometimes this distance measure is also called editing distance. It is widely used in the context of string matching algorithms as needed, for instance, in genome research. On the set $X^{*}$ one considers the three operations $\sigma$ of substituting a symbol, $\iota$ of inserting a symbol and $\delta$ of deleting a symbol. To change a word $x \in X^{*}$ into a word $y \in X^{*}$, one can use a sequence of these operations; the reverse of this sequence will change $y$ into $x$. The length of the shortest such sequence of operations is the Levenshtein

\footnotetext{
2 See [31] for an explanation of the connection between error probability and difference of words.
} 
distance ${ }^{3}$ between $x$ and $y$; the operation $\sigma$ is redundant as it can be simulated by $\iota \delta$. Hence one gets two different distance measures $d_{\sigma, \iota, \delta}$ and $d_{l, \delta}$, both being metrics, which give rise to homeomorphic topologies.

Another idea is proposed in [7]. Let $f: X^{*} \rightarrow \mathbb{R}_{+}$be an injective function such that $f(\varepsilon)=0$. Then the function $d_{f}: X^{*} \times X^{*} \rightarrow \mathbb{R}$ with $d_{f}(x, y)=|f(x)-f(y)|$ for $x, y \in X^{*}$ is a metric. For example, with card $X=q$, let $\alpha: X \rightarrow\{1,2, \ldots, q\}$ be a bijection; for a word $x=a_{1} a_{2} \cdots a_{n} \in X^{*}$ with $a_{i} \in X$ for all $i$, let $f(x)=\sum_{i=1}^{n}(q+1)^{-\alpha\left(a_{i}\right)}$. Then $f$ corresponds to the lexicographical ordering of words in the following sense: $f(x)<f(y)$ if and only if $x \leq$ lex $y$.

In general, a partial order $\leq$ on $X^{*}$ gives rise to a topology Top $_{\leq}$defined by the family $\left\{\right.$Succ $\left._{\leq} u \mid u \in X^{*}\right\}$ as a sub-base of open sets. Among these the prefix topology $T_{\leq_{p}}$ plays a special rôle as the concept of successor coincides with the usual left-to-right reading of words. For the prefix order $\leq_{\mathrm{p}}$ the set of successors of a word $u \in X^{*}$ is the set $u X^{*}$.

For a given partial order, one can derive natural definitions of the notions of density and convexity. For the former, see [30,32]; for the latter, see [1], where the term of continuity is used instead. For additional information see [54,65].

Another interesting method by which a topology could be derived from the comparison of words is analysed in [9] in an abstract setting, not in any way related to orders on words.

\subsection{Topologies from languages}

Let $L \subseteq X^{*}$ be a language (natural or formal) and let $u, v \in X^{*}$. A question raised early on in linguistics was how to quantify the comparison of the rôles played by the words $u$ and $v$ with respect to the language $L$ (see [40]). The set $C_{L}(u)=\left\{(x, y) \mid x, y \in X^{*}, x u y \in L\right\}$ of permitted contexts of $u$ is called the distribution class of $u$. The distribution class of a word can be interpreted as a description of the syntactic or semantic category of the word. Thus one would like to express the topological relation between $u$ and $v$ in terms of a comparison of their distribution classes $C_{L}(u)$ and $C_{L}(v)$. A probabilistic version of these relations was introduced in [33]. Generalizing these thoughts one attempts to compare classes of words, that is, languages. While most of the elementary concepts concerning distribution classes can easily be extended to $\infty$-languages, the topological consequences of such a generalization have not been explored.

Several different proposals for deriving topologies on $X^{*}$ and for equipping $X^{*}$ with a metric, which are based on the language-theoretic concepts, are presented and analysed in $[18,17,20,19,66,49,8]$.

Topologies on $X^{*}$ which are not induced by order relations were considered in [47,48,51]. Further topological properties derived for languages, automata or grammars are studied in [12,64,41,63,27,10,53].

\subsection{Topologies from the multiplicative structure}

In [22] a topology for free groups was introduced (see also [46]). These ideas were generalized to free monoids, that is, to $X^{*}$ in $[44,45]$. At this point we do not know how this work relates to our results.

\section{Review of topologies for finite and infinite words}

It seems that for finite and infinite words one usually only considers the topology related to the prefix order. See [43] for a general introduction. These topologies resemble the ones defined on semirings of formal power series (see [34]).

Topologies on $X^{\infty}$, while needed for a sound definition of $\omega$-words as limits of sequences of $*$-words have not been studied much. As far as we know, the earliest such investigation is reported in $[42,4]$. There, instead of $X^{\infty}$, one considers $(X \cup\{\perp\})^{\omega}$, where $\perp$ is a new symbol such that a $*$-word $w$ is represented by $w \perp^{\omega}$; the topology is then based on the prefix order.

As mentioned above, we are looking for a natural way of extending mappings from finite words to infinite words. The following method, applicable in the case of the prefix topology, will guide the ideas. Let $\varphi: X^{*} \rightarrow X^{*}$ be a mapping which is monotone with respect to $\leq_{\mathrm{p}}$. The natural extension of $\varphi$ to a mapping $\bar{\varphi}: X^{\infty} \rightarrow X^{\infty}$ is then defined by

$$
\bar{\varphi}(\xi)=\sup _{\leq_{\mathrm{p}}}\left\{\varphi(w) \mid w \in X^{*} \wedge w \leq_{\mathrm{p}} \xi\right\}
$$

as shown in Fig. 1. For language-theoretic aspects see $[38,4,58]$.

\subsection{Topologies related to the prefix-limit process}

We consider two topologies which are related to the extension process defined above. The first one is closely related to the topology of the Cantor space $\left(X^{\omega}, \varrho\right)$ where the function $\varrho: X^{\omega} \times X^{\omega} \rightarrow \mathbb{R}$, defined as

$$
\varrho(\xi, \zeta)=\inf \left\{(\operatorname{card} X)^{-|w|} \mid w \in \operatorname{Pref} \xi \cap \operatorname{Pref} \zeta\right\}
$$

for $\xi, \zeta \in X^{\omega}$, is a metric.

\footnotetext{
3 For algorithms to compare strings according to the Levenshtein distance and for applications to DNA-sequencing see [2,11].
} 


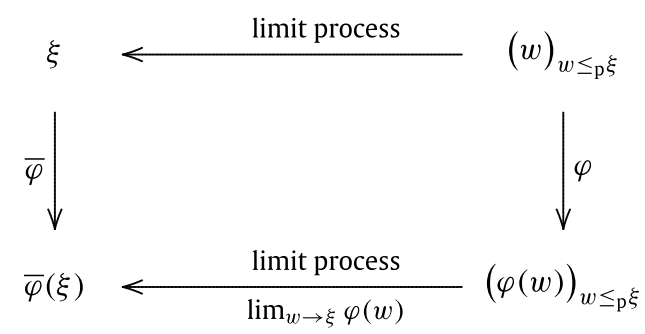

Fig. 1. Extension of a mapping.

\subsubsection{CANTOR topology}

For details regarding the CANTOR topology we refer to [4]. As mentioned above, we introduce a new symbol $\perp$ and represent the words $w \in X^{*}$ by the infinite words $w \perp^{\omega}$. For $\eta, \eta^{\prime} \in X^{\infty}$ one has

$$
\varrho\left(\eta, \eta^{\prime}\right)= \begin{cases}0, & \text { if } \eta=\eta^{\prime}, \\ (\operatorname{card} X)^{1-\operatorname{card}\left(\operatorname{Pref} \eta \cap \operatorname{Pref} \eta^{\prime}\right)}, & \text { otherwise. }\end{cases}
$$

Thus, the space $\left(X^{\infty}, \varrho\right)$ is considered as a subspace of the CANTor space $\left((X \cup\{\perp\})^{\omega}, \varrho\right)$ with all $w \in X^{*}$ as isolated points.

\subsubsection{REDZIEJOWSKI's topology}

A different approach to defining a natural topology on $X^{\infty}$ is proposed in [50]. We refer to this topology as $\tau_{R}$.

Definition 4. Let $W \subseteq X^{*}$ and $F \subseteq X^{\omega}$. We define $\vec{W}=\left\{\xi \mid \xi \in X^{\omega} \wedge \operatorname{Pref} \xi \cap W\right.$ is infinite $\}$ and the $\operatorname{closure~}_{R}(W \cup F)=$ $W \cup F \cup \vec{W}$.

We list a few properties of the topology $\tau_{R}$ (see [50]).

Proposition 5. The topology $\tau_{R}$ on $X^{\infty}$ has the following properties:

(1) The topology $\tau_{R}$ is not a metric topology.

(2) Every subset $F \subseteq X^{\omega}$ is closed.

(3) The topological space $\left(X^{\infty}, \tau_{R}\right)$ is completely regular, hence a HAUSDORFF space.

(4) In contrast to the CANTOR topology, where $\lim _{n \rightarrow \infty} 0^{n} \cdot 1=0^{\omega}$, the sequence $\left(0^{n} \cdot 1\right)_{n \in \mathbb{N}}$ has no limit in $\tau_{R}$, while $\lim _{n \rightarrow \infty} 0^{n}=0^{\omega}$ in both topologies.

\subsection{Adherences}

An operator, very much similar to that of the closure operator in the CANTOR topology, called adherence, (or ls-operator) was introduced to formalize the transition from finite to infinite words (see $[57,61,38,42,4,58,59,26,28,36,15,23-25,55,62]$ ). Adherence is defined as an operator on languages as follows.

Definition 6. The adherence of a language $W \subseteq X^{*}$ is the set Adh $W=\left\{\xi \mid \xi \in X^{\omega} \wedge\right.$ Pref $\xi \subseteq$ Pref $\left.W\right\}$.

An $\omega$-word $\xi$ is an element of Adh $W$ if and only if, for all $v \leq_{\mathrm{p}} \xi$, the set $W \cap v X^{*}$ is infinite.

\subsubsection{Adherence and topologies}

The following facts connect the concept of adherence with the closure operator in the CANToR topology of $X^{\infty}$.

Proposition 7. Let $W \subseteq X^{*}$ and $F \subseteq X^{\omega}$. The CANTOR topology on $X^{\infty}$ has the following properties:

(1) The adherence Adh $W$ is the set of cluster points of $W$.

(2) The closure of $W \cup F$ is the set $W \cup \operatorname{Adh}(W \cup \operatorname{Pref} F)$.

\subsubsection{Adherences as limits}

Given the connection between adherence and closure, it is not surprising that adherence can be viewed as a kind of limit.

Proposition 8. Let $\varphi: X^{*} \rightarrow X^{*}$ be a mapping which is monotone with respect to $\leq_{\mathrm{p}}$ and let $\xi \in X^{\omega}$. If the set $\varphi$ (Pref $\left.\xi\right)$ is infinite then $\{\bar{\varphi}(\xi)\}=\operatorname{Adh}\left\{\varphi(w) \mid w<_{\mathrm{p}} \xi\right\}$.

Definition 9. A mapping $\varphi: X^{*} \rightarrow Y^{*}$ is said to be totally unbounded if $\varphi(W)$ is infinite whenever $W \subseteq X^{*}$ is infinite.

Theorem $10([57,61,38,4,58])$. If $\varphi: X^{*} \rightarrow Y^{*}$ is totally unbounded and monotone with respect to $\leq_{\mathrm{p}}$ then $\bar{\varphi}($ Adh $W)=$ $\operatorname{Adh} \varphi(W)$ and $\bar{\varphi}^{-1}(\operatorname{Adh} W)=\operatorname{Adh} \varphi^{-1}(\operatorname{Pref} W)$. 


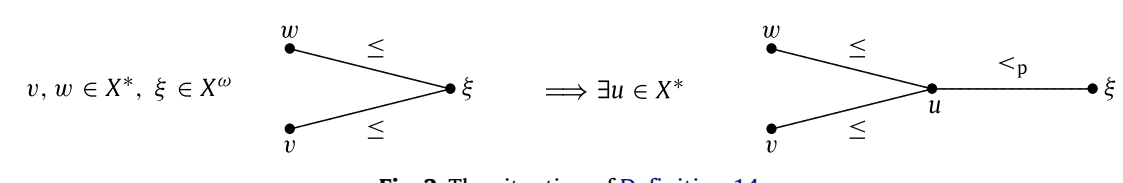

Fig. 2. The situation of Definition 14.

\section{Extending partial orders}

As mentioned above the need to consider partial orders different from the prefix order arose from the following general consideration in [6]: We needed to make a statement about the density of a certain kind of language with respect to all kinds of reasonable topologies; the prefix topology would have been just one special, albeit natural, case. Moreover, we needed a topologically well-founded transition between $X^{*}$ and $X^{\infty}$ which did not rely on the artifact of a padding symbol like $\perp$ considered before. Therefore, in this section we consider extensions of partial orders $\leq$ on $X^{*}$ to the set $X^{\infty}$. Since we want the infinite words to be limits of sequences of finite words, we make them maximal elements in the extended order.

Definition 11. Let $\leq$ be a partial order on $X^{*}$. The relation $\preceq$ on $X^{\infty} \times X^{\infty}$ defined by

$$
\eta \preceq \eta^{\prime} \Longleftrightarrow \begin{cases}\eta \leq \eta^{\prime}, & \text { if } \eta, \eta^{\prime} \in X^{*}, \\ \eta=\eta^{\prime}, & \text { if } \eta, \eta^{\prime} \in X^{\omega}, \\ \exists v\left(v \in X^{*} \wedge \eta \leq v<_{\mathrm{p}} \eta^{\prime}\right), & \text { if } \eta \in X^{*} \text { and } \eta^{\prime} \in X^{\omega}\end{cases}
$$

is called the extension of $\leq$.

In order to show that $\preceq$ as defined in Definition 11 is indeed a partial order on $X^{\infty}$ with all $\xi \in X^{\omega}$ as maximal elements it suffices to verify that $\preceq$ is transitive on $X^{\infty}$. This follows from Eq. (6) and the transitivity of $\leq$ on $X^{*}$.

A partial order $\preceq$ on $X^{\infty}$ derived from a partial order $\leq$ on $X^{*}$ according to Eq. (6) is called an extended partial order; when there is no risk of confusion, we denote the original partial order and its extension by the same symbol $\leq$.

A characteristic property of extended partial orders is that, for $w \in X^{*}$ and $\xi \in X^{\omega}$, the inequality $w<_{\mathrm{p}} \xi$ implies $w<\xi$. From the third case of Definition 11 one concludes:

Remark 12. Let $\leq$ be an extended partial order on $X^{\infty}$. For all $\xi \in X^{\omega}$ and all $w \in X^{*}$, if $w<_{\mathrm{p}} \xi$ then $w \leq \xi$. Thus $\operatorname{Pref} \xi \subseteq \operatorname{Pred}_{\leq} \xi$.

Thus, from Definition 11, we obtain a relation between the sets $\mathbf{B}_{w}=\left\{\eta \mid \eta \in X^{\infty} \wedge w \leq \eta\right\}=$ Succ $_{\leq} w$ and Pref $\xi$ for $w \in X^{*}$ and $\xi \in X^{\omega}$.

Proposition 13. Let $\xi \in X^{\omega}, w \in X^{*}$, and let $\leq$ be an extended partial order on $X^{\infty}$. Then $\xi \in \mathbf{B}_{w}$, that is, $w \leq \xi$, if and only if Pref $\xi \cap \mathbf{B}_{w} \neq \emptyset$.

Proof. If $w \leq \xi$ there is a $u<_{\mathrm{p}} \xi$ such that $w \leq u$. Conversely, if Pref $\xi \cap \mathbf{B}_{w} \neq \emptyset$ then there is a $u \in X^{*}$ such that $u<_{\mathrm{p}} \xi$ and $w \leq u$; hence $w \leq \xi$ by Definition 11 .

Definition 14. An extended partial order $\leq$ is said to be confluent if, for all $w, v \in X^{*}$ and all $\xi \in X^{\omega}$ with $w, v \leq \xi$, there is a word $u \in X^{*}$ such that $w, v \leq u$ and $u<_{\mathrm{p}} \xi$.

The situation in Definition 14 is illustrated in Fig. 2. For a confluent extended partial order $\leq$ we have either $\mathbf{B}_{w} \cap \mathbf{B}_{v}=\emptyset$ or $u X^{\omega} \subseteq \mathbf{B}_{w} \cap \mathbf{B}_{v}$ for some $u \in X^{*}$.

Example 15. Let $X=\{a, b\}$. The extension of the suffix relation $\leq_{\mathrm{s}}$ is not confluent. We have $a, b \leq_{\mathrm{s}}(a b)^{\omega}$ but there is no $u \in\{a, b\}^{*}$ such that $a, b \leq_{s} u$.

By Corollary 20 and Example 21, the extensions of several highly relevant partial orders are indeed confluent. For extended partial orders we obtain the following equivalence.

Lemma 16. Let $\leq$ be an extended partial order. The relation $\leq$ is confluent if and only if $\mathbf{B}_{w} \cap \mathbf{B}_{v}=\bigcup_{w \in X^{*}} \mathbf{B}_{u}$ for all $w, v \in X^{*}$.

Proof. For all $u, v \in X^{*}$ one has $v \leq u$ if and only if $\mathbf{B}_{u} \subseteq \mathbf{B}_{v}$ for any extended partial order $\leq$. This proves the inclusion $\supseteq$.

Now assume that $\leq$ is confluent. We prove the converse inclusion. Let $\eta \in \mathbf{B}_{w} \cap \mathbf{B}_{v}$, that is, $w, v \leq \eta$. If $\eta \in X^{*}$ then $\eta \in \mathbf{B}_{\eta} \subseteq \bigcup_{w, v \leq u} \mathbf{B}_{u}$. If $\eta \in X^{\omega}$, in view of Definition 14 there is a $u \in X^{*}$ with $w, v \leq u<{ }_{\mathrm{p}} \eta$. This yields $\eta \in \mathbf{B}_{u}$.

To prove the converse implication consider $w, v \in X^{*}$ and $\xi \in X^{\omega}$ with $w, v \leq \xi$. Then $\xi \in \mathbf{B}_{w} \cap \mathbf{B}_{v}=\bigcup_{w, v \leq u} \mathbf{B}_{u}$. Consequently there is a $u \in X^{*}$ such that $w, v \leq u$ and $\xi \in \mathbf{B}_{u}$, that is, $u<_{\mathrm{p}} \xi$.

Lemma 16 gives a representation of $\mathbf{B}_{w} \cap \mathbf{B}_{v}$ as a (possibly empty) union of sets $\mathbf{B}_{u}$ with $u \in X^{*}$. In a minimal representation of $\mathbf{B}_{w} \cap \mathbf{B}_{v}$ as a union $\bigcup \underset{w, v \leq u}{u \in W} \mathbf{B}_{u}$ of sets where $W \subseteq X^{*}$, the index set $W$ can be finite or infinite, even for the same relation $\leq$.

Example 17. We consider the infix order $\leq_{i}$.

(1) If $X=\{0,1\}$ then $\mathbf{B}_{0} \cap \mathbf{B}_{1}=\mathbf{B}_{01} \cup \mathbf{B}_{10}$, and the union is finite, whereas the minimal representation $\mathbf{B}_{01} \cap \mathbf{B}_{10}=$ $\bigcup_{n \geq 1} \mathbf{B}_{01^{n}{ }_{0}} \cup \mathbf{B}_{10^{n_{1}}}$ is an infinite union.

(2) If we consider $\mathbf{B}_{0} \cap \mathbf{B}_{1}$ over the ternary alphabet $X=\{0,1,2\}$ then a minimal representation is $\mathbf{B}_{0} \cap \mathbf{B}_{1}=\bigcup_{n \geq 0} \mathbf{B}_{02^{n} 1} \cup$

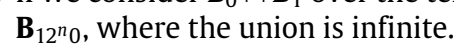

Neither $\mathbf{B}_{01} \cap \mathbf{B}_{10}$ nor, in the ternary case, $\mathbf{B}_{0} \cap \mathbf{B}_{1}$ can be represented as finite unions. 


\subsection{Prefix-based partial orders}

Intuitively, taking limits of words implies that one moves from prefixes to prefixes; hence the pre-dominance of considerations based on the prefix order. While we shall not dwell on this point in the present paper, it is far less intuitive what a topology on words would look like if one took away the European way of reading words from left to right. In this section we consider topologies from partial orders which are compatible with the prefix order. Hence, ideas derived for the latter can be adequately generalized. We investigate particular cases of confluent extended partial orders. Several prominent instances of such orders are given in Example 21.

Definition 18. A partial order $\leq$ on $X^{*}$ is said to be prefix-based if, for all $w, v \in X^{*}, w \leq_{\mathrm{p}} v$ implies $w \leq v$,

Lemma 19. A partial order $\leq$ on $X^{*}$ is prefix-based if and only if, for all $w, v, u \in X^{*}, w \leq v$ and $v \leq_{\mathrm{p}} u$ imply $w \leq u$.

Proof. Let $\leq$ be prefix-based and let $w \leq v$ and $v \leq_{\mathrm{p}} u$. Then $v \leq u$ and, since $\leq$ is transitive, we get $w \leq u$.

Conversely, if $w \leq v$ and $v \leq_{\mathrm{p}} u$ imply $w \leq u$, we choose $w=v$ and obtain that $w \leq_{\mathrm{p}} u$ implies $v \leq \bar{u}$.

Corollary 20. If a partial order $\leq$ on $X^{*}$ is prefix-based then its extension to $X^{\infty}$ is confluent.

Proof. Assume $w, v \leq \xi$ for $w, v \in X^{*}$ and $\xi \in X^{\omega}$. According to Eq. (6) there are $u_{w}, u_{v} \in X^{*}$ such that $w \leq u_{w}<_{\mathrm{p}} \xi$ and $v \leq u_{v}<_{\mathrm{p}} \xi$. Without loss of generality let $u_{w} \leq_{\mathrm{p}} u_{v}$. Since $\leq$ is prefix-based, this implies also $w \leq u_{v}<_{\mathrm{p}} \xi$; hence $\leq$ is confluent.

Example 21. The following partial orders are prefix-based:

(1) infix order $\leq_{\mathrm{i}}$,

(2) embedding (or shuffle) order $\leq_{e}$,

(3) quasi-lexicographical order $\leq_{\mathrm{q}-\mathrm{lex}}$, and

(4) lexicographical order $\leq_{\text {lex }}$.

When $\leq=\leq_{\mathrm{p}}$ the resulting topology $\tau_{\leq_{\mathrm{p}}}$ on $X^{\infty}$ is a ScoTt topology (see [56]), that is, every directed family $w_{0} \leq_{\mathrm{p}} \cdots w_{i} \leq_{\mathrm{p}}$ $w_{i+1} \leq_{\mathrm{p}} \cdots$ has a least upper bound. The partial orders considered above do not have this property.

Consider, for example, the directed family $0 \leq \cdots \leq 0^{i} \leq 0^{i+1} \leq \cdots$ where $\leq$ is a partial order. When $\leq=\leq{ }_{\mathrm{p}}$, the $\omega$-word $0^{\omega}$ is the unique (and "natural") upper bound. On the other hand, when $\leq$ is any one of the relations considered above, in addition to $0^{\omega}$, also $\prod_{i=0}^{\infty} 0^{i} \cdot 1$ is an upper bound.

For prefix-based relations $\leq$ we have a connection between $\leq$ and the prefix relation similar to Proposition 13 .

Proposition 22. Let $\xi \in X^{\omega}, w \in X^{*}$, and let $\leq$ be the extension of a prefix-based partial order. Then $w \leq \xi$ if and only if $\operatorname{Pref} \xi \backslash \mathbf{B}_{w}$ is finite.

Proof. If $w \leq \xi$ there is a $u<_{\mathrm{p}} \xi$ such that $w \leq u$. Lemma 19 implies that, for all $v \in X^{*}$ with $u \leq_{\mathrm{p}} v<_{\mathrm{p}} \xi$, $w \leq v$. Hence, if $y \in \operatorname{Pref} \xi \backslash \mathbf{B}_{w}$, then $y<_{\mathrm{p}} u$; thus Pref $\xi \backslash \mathbf{B}_{w}$ is finite.

Conversely, if Pref $\xi \backslash \mathbf{B}_{w}$ is finite then Pref $\xi \cap \mathbf{B}_{w} \neq \emptyset$; the assertion follows by Proposition 13.

\section{Quasi-right topologies}

In order to relate the topologies to a limit process approaching infinite words by finite ones we should require that an infinite word $\xi \in X^{\omega}$ not be an isolated point in the topology $\tau_{\leq}$derived from $\leq$. This is in contrast to the situation in the right topology $\alpha_{\leq}$on $X^{\infty}$.

To this end, we consider quasi-right topologies on the set $X^{\infty}$ partially ordered by some relation $\leq$. In contrast to the right topology $\boldsymbol{\alpha}_{\leq}$the quasi-right topology $\tau_{\leq}$on $X^{\infty}$ derived from the extended partial order $\leq$ is generated by the sub-basis $\left(\mathbf{B}_{w}\right)_{w \in X^{*}}$ where $\mathbf{B}_{w}=\left\{\eta \mid \eta \in X^{\infty} \wedge w \leq \eta\right\}$. Thus we do not include the sets $\mathbf{B}_{\xi}$ for $\xi \in X^{\omega}$ in the class of open sets.

For extended partial orders, Definition 11 yields the following representation:

$$
\mathbf{B}_{w}=\left\{v \mid v \in X^{*} \wedge w \leq v\right\} \cdot\left(\{\varepsilon\} \cup X^{\omega}\right) .
$$

Similarly to the right topology $\boldsymbol{\alpha}_{\leq}$, for $w \in X^{*}$, the set $\mathbf{B}_{w}$ is the smallest open set containing $w$, and, since the family $\left(\mathbf{B}_{w}\right)_{w \in X^{*}}$ is countable, the topology $\tau_{\leq}$has the countable basis $\left\{\bigcap_{i=1}^{n} \mathbf{B}_{w_{i}} \mid n \in \mathbb{N} \wedge w_{i} \in X^{*}\right.$ for $\left.i=1, \ldots, n\right\}$.

From Lemma 16, we obtain a necessary and sufficient condition as to when the family $\left(\mathbf{B}_{w}\right)_{w \in X^{*}}$ is a basis.

Proposition 23. The family $\left(\mathbf{B}_{w}\right)_{w \in X^{*}}$ is a basis of the topology $\tau_{\leq}$if and only if the extended partial order $\leq$is confluent.

Proposition 24. Let $\leq$ be an extended partial order on $X^{\infty}$. Then, for $\xi \in X^{\omega}$, one has $\mathbf{B}_{\xi}=\{\xi\}$, and $\mathbf{B}_{\xi}$ is not open in $\tau_{\leq}$. If, moreover, the order $\leq$ is confluent, then no non-empty subset $F \subseteq X^{\omega}$ is open.

Proof. By definition, $\mathbf{B}_{\xi}=\{\xi\}$. Assume that $\{\xi\}$ is open. Then $\{\xi\}$ contains a non-empty basis set $\bigcap_{i=1}^{n} \mathbf{B}_{w_{i}}$. Hence $\xi \in \mathbf{B}_{w_{i}}$, for all $i=1, \ldots, n$. Consequently, for every $i=1$, there is a prefix $u_{i}<_{\mathrm{p}} \xi$ such that $w_{i} \leq u_{i}$. Let $u_{1}$ be the longest of these prefixes. Then, according to Eq. (7), every $\mathbf{B}_{w_{i}}$ contains the set $u_{1} \cdot X^{\omega}$. Hence $u_{1} X^{\omega} \subseteq\{\xi\}$, a contradiction.

Now, let $\leq$ be confluent and let $F$ be a non-empty open subset of $X^{\omega}$. By Proposition 23, $\mathbf{B}_{w} \subseteq F$ for some $w \in X^{*}$. This contradicts $F \subseteq X^{\omega}$.

The next example shows that the hypothesis for $\leq$ to be confluent is indeed essential.

Example 25. Consider $X=\{0,1\}$ and the suffix order $\leq_{s}$. Then $\mathbf{B}_{0} \cap \mathbf{B}_{1}=X^{\omega} \backslash\left\{0^{\omega}, 1^{\omega}\right\} \subseteq X^{\omega}$ is open. 


\subsection{Accumulation points and cluster points}

In this part we use the fact that $\mathbf{B}_{w}$ is the smallest open set containing $w \in X^{*}$ to describe the accumulation and cluster points in the topology $\tau_{\leq}$in greater detail. As an immediate consequence, we obtain a result on finite words.

Lemma 26. Let $w \in X^{*}$ and $M \subseteq X^{\infty}$.

(1) $w$ is an accumulation point of $M$ with respect to $\tau_{\leq}$if and only if $\mathbf{B}_{w} \cap(M \backslash\{w\}) \neq \emptyset$.

(2) $w$ is a cluster point of $M$ with respect to $\tau_{\leq}$if and only if $\mathbf{B}_{w} \cap M$ is infinite.

For infinite words we obtain the following.

Lemma 27. Let $\xi \in X^{\omega}$ and $M \subseteq X^{\infty}$.

(1) $\xi$ is an accumulation point of $M$ with respect to $\tau_{\leq}$if and only if Pref $\xi \subseteq\left\{v \mid \mathbf{B}_{v} \cap(M \backslash\{\xi\}) \neq \emptyset\right\}$.

(2) $\xi$ is a cluster point of $M$ with respect to $\tau_{\leq}$if and only if Pref $\xi \subseteq\left\{v \mid \operatorname{card}\left(\mathbf{B}_{v} \cap M\right) \geq \aleph_{0}\right\}$.

Proof. If $\xi$ is an accumulation point of $M$ then $M^{\prime} \cap(M \backslash\{\xi\}) \neq \emptyset$ for every open set $M^{\prime}$ containing $\xi$. This holds, in particular, for every basis set $\mathbf{B}_{v}$ with $v<_{\mathrm{p}} \xi$.

Conversely, let Pref $\xi \subseteq\left\{v \mid \mathbf{B}_{v} \cap(M \backslash\{\xi\}) \neq \emptyset\right\}$, and let $M^{\prime}$ be an open set with $\xi \in M^{\prime}$. Then there is a basis set $\mathbf{B}_{w} \subseteq M^{\prime}$ containing $\xi$. Thus, $w \leq \xi$ and, according to Definition 11, there is a $v<_{\mathrm{p}} \xi$ such that $w \leq v$. Thus $\mathbf{B}_{v} \cap(M \backslash\{\xi\}) \neq \emptyset$. Since $w \leq v$, we obtain $\xi \in \mathbf{B}_{v} \subseteq \mathbf{B}_{w} \subseteq M^{\prime}$. Consequently, $M^{\prime} \cap(M \backslash\{\xi\}) \neq \emptyset$.

The proof of the second part is obtained analogously, replacing the condition of $\mathbf{B}_{v} \cap(M \backslash\{\xi\}) \neq \varnothing$ by that of $\operatorname{card}\left(\mathbf{B}_{v} \cap M\right) \geq \aleph_{0}$.

Now Eq. (5) yields the following characterisation of the closure $\mathrm{cl}_{\leq}$.

Corollary 28. Let $\leq$ be an extended partial order on $X^{\infty}$ and, for $M \subseteq X^{\infty}$, let $\bar{M}=\left\{w \mid w \in X^{*} \wedge \mathbf{B}_{w} \cap M \neq \emptyset\right\}$. Then

$$
\mathrm{cl}_{\leq}(M)=\bar{M} \cup\left\{\xi \mid \xi \in X^{\omega} \wedge \operatorname{Pref} \xi \subseteq \bar{M}\right\} .
$$

The following example shows that in our topologies - unlike metric topologies - accumulation points and cluster points, even in $X^{\omega}$, can be different.

Example 29. Consider $X=\{0,1\}$ and the quasi-lexicographic order $\leq_{\mathrm{q}-l e x}$. All non-empty open sets contain $1^{\omega}$. Thus every $\xi \in\{0,1\}^{\omega} \backslash\left\{1^{\omega}\right\}$ is an accumulation point of the set $M=\left\{1^{\omega}\right\}$. But $M$ has no cluster points.

Definition 30. A partial order $\leq$ on $X^{\infty}$ is well-founded if, for every $w \in X^{*}$, the set $\operatorname{Pred}_{\leq} w$ of predecessors of $w$ is finite,

Theorem 31. Let $\leq$ be a well-founded prefix-based partial order on $X^{\infty}$, let $W \subseteq X^{*}$ and let $\xi \in X^{\omega}$. Then $\xi$ is an accumulation point of $W$ if and only if it is a cluster point of $W$.

Proof. By Remark 2 every cluster point of $W$ is an accumulation point of $W$. For the converse, we use Lemmata 26 and 27 to show that, if, for all $v<_{\mathrm{p}} \xi, \mathbf{B}_{v} \cap W \neq \emptyset$ then, for all $v<_{\mathrm{p}} \xi, \mathbf{B}_{v} \cap W$ is infinite.

Assume that, for all $v<_{\mathrm{p}} \xi, \mathbf{B}_{v} \cap W \neq \emptyset$ and consider a word $u$ with $v<_{\mathrm{p}} u<_{\mathrm{p}} \xi$. Since $\leq$ is prefix-based, we have also $v<u$ and thus $\mathbf{B}_{v} \cap W \supseteq \mathbf{B}_{u} \cap W$. This shows that $\left(\mathbf{B}_{u} \cap W\right)_{u<p} \xi$ is an infinite descending family of non-empty sets.

If $w \in \bigcap_{u<\mathrm{p} \xi} \mathbf{B}_{u} \cap W$ then $u \leq w$ for all $u \in \operatorname{Pref} \xi$ which contradicts the fact that $\leq$ is well-founded. Consequently, $\left(\mathbf{B}_{u} \cap W\right)_{u<p} \xi$ is an infinite descending family of non-empty sets having an empty intersection. Therefore, all $\mathbf{B}_{u} \cap W$ are infinite.

We conclude this subsection with two examples which show that the assumptions regarding the partial order $\leq$ in Theorem 31 are essential.

Example 32. The lexicographical order $\leq_{\text {lex }}$ is not well-founded but prefix-based. Consider the language $W=\{11\} \subseteq$ $\{0,1\}^{*}$. Then the infinite word $1 \cdot 0^{\omega}$ is an accumulation point of $W$. Since $W$ is finite, it cannot have cluster points.

Example 33. The suffix order $\leq_{s}$ is well-founded but not prefix-based. Let again $X=\{0,1\}$ and consider the language $W=\{0\} \cup 1^{*} \cdot 101 \cdot 1^{*}$ and the infinite word $\xi=0 \cdot 1^{\omega}$. Here $\mathbf{B}_{w} \cap W \neq \emptyset$ for all $w \in \operatorname{Pref} \xi=\{\varepsilon\} \cup 0 \cdot 1^{*}$ and $\mathbf{B}_{0} \cap W$ is finite. 


\subsection{Adherences related to the topologies $\tau_{\leq}$}

It is interesting to note that that the closure operator $\mathrm{cl}_{\leq}$of the topology $\tau_{\leq}$is closely related to the language-theoretical operation of adherence. Adherence (or ls-limit) was first introduced for the prefix relation $\leq_{p}$ (see $[57,61,38,42,4,58,59]$ ), and then in [16] for the infix order $\leq_{i}$.

In this section we define the operation of adherence for arbitrary extended partial orders $\leq$ and we prove its relation to the corresponding closure operation $\mathrm{cl}_{\leq}$. Moreover we show that for prefix-based partial orders adherence can be expressed with the aid of the prefix order.

For notational convenience, given a partial order $\leq$ on $\mathcal{X}$, we define a relation, also denoted by $\leq$, on $2^{x}$ as follows: Let $M, M^{\prime} \subseteq X$. Then $M \leq M^{\prime}$ if and only if for every $x \in M$ there is an $x^{\prime} \in M^{\prime}$ such that $x \leq x^{\prime}$.

Proposition 34. The relation $\leq$ on $2^{x}$ has the following properties:

(1) $\leq$ is reflexive and transitive.

(2) $\leq$ is not necessarily anti-symmetric.

(3) $M \subseteq M^{\prime}$ implies $M \leq M^{\prime}$.

(4) $\varnothing \leq M$ for all $M \subseteq X$.

(5) Let I be a set and, for $i \in I$, let $M_{i}, M_{i}^{\prime} \subseteq X$ such that $M_{i} \leq M_{i}^{\prime}$. Then $\bigcup_{i \in I} M_{i} \leq \bigcup_{i \in I} M_{i}^{\prime}$.

(6) With $X=X^{\infty}$, let $\leq$ be an extended partial order, let $M \subseteq X^{*}$ and $M^{\prime} \subseteq X^{\infty}$. Then $M \leq M^{\prime}$ if and only if $M \subseteq\{w \mid$ $\left.\mathbf{B}_{w} \cap M^{\prime} \neq \emptyset\right\}$.

Proof. Assertions (1)-(5) are direct consequences of the definition. For (6) one uses Eq. (7).

By Proposition 34(6), in the particular case of the prefix order $\leq_{\mathrm{p}}$ and subsets $W \subseteq X^{*}, M^{\prime} \subseteq X^{\infty}$, one has $W \leq_{\mathrm{p}} M^{\prime}$ if and only if $W \subseteq \operatorname{Pref} M^{\prime}$.

For extended partial orders we obtain the following properties.

Lemma 35. Let $\leq$ be an extended partial order on $X^{\infty}$, let $\xi \in X^{\omega}$, and let $M \subseteq X^{\infty}$. Then $\operatorname{Pred}_{\leq} \xi \leq M$ if and only if Pref $\xi \leq M$.

Proof. By Remark 12, Pref $\xi \subseteq \operatorname{Pred}_{\leq} \xi$; hence, if $\operatorname{Pred}_{\leq} \xi \leq M$ then Pref $\xi \leq M$. To prove the converse implication, consider $v \leq \xi$. Then there is a $u<_{\mathrm{p}} \xi$ such that $v \leq u$. As Pref $\xi \leq M$, there is a $w \in M$ with $u \leq w$. As $\leq$ is transitive, one has $v \leq w$.

Lemma 36. Let $\leq$ be an extended partial order on $X^{\infty}$. If $W \subseteq X^{*}$ and $F \subseteq X^{\omega}$ then $W \leq F$ if and only if $W \leq \operatorname{Pref} F$.

Proof. $W \leq F$ holds true if and only if, for all $w \in W$, there is a $\xi \in F$ such that $w \leq \xi$. The latter is equivalent to the existence of a prefix $v \in \operatorname{Pref} \xi$ such that $w \leq v$.

In particular, one has $\mathbf{B}_{v} \cap F \neq \emptyset$ if and only if $\mathbf{B}_{v} \cap \operatorname{Pref} F \neq \emptyset$.

Theorem 37. Let $M \subseteq X^{\infty}, W \subseteq X^{*}$ and $F \subseteq X^{\omega}$, and let $\leq$ be an extended partial order on $X^{\infty}$. Then $M \leq W \cup F$ if and only if $M \cap X^{\omega} \subseteq F$ and $M \cap X^{*} \leq W \cup \operatorname{Pref} F$.

Proof. $M \leq W \cup F$ implies that $M \cap X^{\omega} \leq W \cup F$ and $M \cap X^{*} \leq W \cup F$. As $\leq$ is the identity on $X^{\omega}, M \cap X^{\omega} \subseteq F$ follows. Split $M \cap X^{*}$ into $M_{W} \cup M_{F}$ such that $M_{W} \leq W$ and $M_{F} \leq F$. Then, by Lemma 36, $M_{F} \leq \operatorname{Pref} F$; hence $M \cap X^{*} \leq W \cup \operatorname{Pref} F$ by Proposition 34(5).

Conversely, let $M \cap X^{\omega} \subseteq F$ and $M \cap X^{*} \leq W \cup \operatorname{Pref} F$. Again the splitting argument for $M \cap X^{*}$ and the recombination of the three parts $M \cap X^{\omega}, M_{W}$ and $M_{F}$ prove the assertion using Lemma 36 and Proposition 34(5).

We now define the adherence with respect to arbitrary extended partial orders. To do so we follow the pattern used for the prefix order.

Definition 38. Let $\leq$ be an extended partial order on $X^{\infty}$ and let $W \subseteq X^{*}$. Then the set

$$
\operatorname{Adh}_{\leq} W=\left\{\xi \mid \xi \in X^{\omega} \wedge \forall v \in \operatorname{Pred}_{\leq} \xi \exists w \in W v \leq w\right\}
$$

is the $\leq$-adherence of $W$.

Remark 39. $\operatorname{Adh}_{\leq} W=\left\{\xi \mid \xi \in X^{\omega} \wedge \operatorname{Pred}_{\leq} \xi \leq W\right\}$.

Proposition 40. If $\leq$ is an extended partial order then

$$
\operatorname{Adh}_{\leq} W=\left\{\xi \mid \xi \in X^{\omega} \wedge \operatorname{Pref} \xi \leq W\right\} .
$$

Proof. This follows from Lemma 35.

Lemma 41. Let $\leq$ be an extended partial order on $X^{\infty}$ and $W \subseteq X^{*}$.

(1) $\mathrm{Adh}_{\leq} W$ is the set of accumulation points of $W$ in $X^{\omega}$.

(2) If $\leq$ is well-founded and prefix-based then $\mathrm{Adh}_{\leq} W$ is the set of cluster points of $W$. 
Proof. Let $\xi \in X^{\omega}$. In view of the equivalence of $v \leq w$ and $w \in \mathbf{B}_{v}$ we have Pref $\xi \subseteq\left\{v \mid \mathbf{B}_{v} \cap W \neq \emptyset\right\}$ if and only if $\operatorname{Pref} \xi \leq W$. Now Proposition 40 proves the first assertion. Assertion (2) follows from (1) and Theorem 31.

Now we can prove the result as announced.

Theorem 42. Let $W \subseteq X^{*}, F \subseteq X^{\omega}$, and let $\leq$ be an extended partial order on $X^{\infty}$. Then the closure of $W \cup F$ in the topology $\tau_{\leq}$ satisfies

$$
\mathrm{cl}_{\leq}(W \cup F)=\operatorname{Pred}_{\leq}(W \cup F) \cup \operatorname{Adh}_{\leq}(W \cup \operatorname{Pref} F) .
$$

Proof. By Corollary 28 one has

$$
\mathrm{cl}_{\leq}(W \cup F)=\left\{v \mid v \in X^{*} \wedge \mathbf{B}_{v} \cap(W \cup F) \neq \emptyset\right\} \cup\left\{\xi \mid \xi \in X^{\omega} \wedge \operatorname{Pref} \xi \subseteq\left\{v \mid \mathbf{B}_{v} \cap(W \cup F) \neq \emptyset\right\}\right\} .
$$

Observe that $\left\{v \mid v \in X^{*} \wedge \mathbf{B}_{v} \cap(W \cup F) \neq \emptyset\right\}=\operatorname{Pred}_{\leq}(W \cup F)$. Lemma 36 shows that the conditions $\mathbf{B}_{v} \cap(W \cup F) \neq \emptyset$ and $\mathbf{B}_{v} \cap(W \cup \operatorname{Pref} F) \neq \varnothing$ are equivalent. Thus

$$
\left\{\xi \mid \xi \in X^{\omega} \wedge \operatorname{Pref} \xi \subseteq\left\{v \mid \mathbf{B}_{v} \cap(W \cup F) \neq \emptyset\right\}\right\}=\left\{\xi \mid \xi \in X^{\omega} \wedge \forall v\left(v \leq_{\mathrm{p}} \xi \rightarrow v \in W \cup \operatorname{Pref} F\right)\right\},
$$

and the assertion is proved.

For the infix order, Dare and Siromoney [16] obtained the identity $\mathrm{cl}_{\leq_{\mathrm{i}}}(W)=\operatorname{Inf}(W \cup F) \cup \operatorname{Adh}_{\leq_{\mathrm{i}}}(W \cup \operatorname{Inf} F)$ where $\operatorname{Inf} M=\left\{v \mid v \in X^{*} \wedge \exists \eta\left(\eta \in M \wedge v \leq_{\mathrm{i}} \eta\right)\right\}$. As Pred $_{\leq_{\mathrm{i}}}=\operatorname{Inf}$ the result of [16] is a special case of Theorem 42.

Corollary 43. Let $\leq$ be an extended partial order on $X^{\infty}$, and let $W \subseteq X^{*}$. Then $\operatorname{Adh}_{\leq} W=\mathrm{cl}_{\leq}(W) \cap X^{\omega}$.

\subsection{Limits of sequences}

We investigate general properties of the topological spaces $\tau_{\leq}$in connection with the language-theoretical operation adherence. As mentioned before we want to study limits of sequences $w_{0}<\cdots<w_{j}<w_{j+1}<\cdots$ in the topology $\tau_{\leq}$.

Recall that a point $\eta \in X^{\infty}$ is in the limit of the sequence $\left(w_{j}\right)_{j \in \mathbb{N}}$ if and only if $w_{j} \leq \eta$ for almost all $j \in \mathbb{N}$. Thus, if $w_{i} \neq w_{j}$ for $i \neq j$, the set of limit points $\lim w_{j}$ is a subset of the set of cluster points of $\left\{w_{j} \mid j \in \mathbb{N}\right\}$.

Lemma 44. Let $w_{0}<w_{1}<\cdots<w_{j}<\cdots$ be an infinite family of words, and let the partial order $\leq$ be well-founded. Then $\lim \left(w_{j}\right)_{j \in \mathbb{N}}=\operatorname{Adh}_{\leq}\left\{w_{j} \mid j \in \mathbb{N}\right\}$.

Proof. As $\leq$ is well-founded, no limit point of $\left(w_{j}\right)_{j \in \mathbb{N}}$ can be a finite word. The inclusion $\lim \left(w_{j}\right)_{j \in \mathbb{N}} \subseteq \mathrm{cl}_{\leq}\left\{w_{j} \mid j \in \mathbb{N}\right\}$ follows from Theorem 1, because the topology $\tau_{\leq}$has a countable basis, and from Corollary 43.

Conversely, let $\xi \in \mathrm{cl}_{\leq}\left\{w_{j} \mid j \in \mathbb{N}\right\} \cap X^{\omega}=\operatorname{Adh}_{\leq}\left\{w_{j} \mid j \in \mathbb{N}\right\}$. Then, according to Corollary 28, for every open set $M$ containing $\xi$ there is a $j_{0} \in \mathbb{N}$ such that $w_{j_{0}} \in M$. Without loss of generality, we may assume $M=\bigcap_{i=1}^{n} \mathbf{B}_{v_{i}}$ to be a basis set. Thus $v_{i} \leq w_{j_{0}}$ for $i=1, \ldots, n$. Now the assumption $w_{0}<w_{1}<\cdots<w_{j}<\cdots$ shows that $w_{j} \in \mathbf{B}_{v_{i}}$ for all $i=1, \ldots, n$ and $j \geq j_{0}$; hence $\xi \in \lim \left(w_{j}\right)_{j \in \mathbb{N}}$.

\section{The topology on $X^{\omega}$ induced by $\tau_{\leq}$}

In this section we briefly investigate the topologies $\tau_{\leq}^{(\omega)}$ on the space of infinite words $X^{\omega}$ which are induced by the quasi-right topologies $\tau_{\leq}$on $X^{\infty}$. These topologies are defined by the sub-basis $\left(\mathbf{E}_{w}\right)_{w \in X^{*}}$ where

$$
\mathbf{E}_{w}=\left\{\xi \mid \xi \in X^{\omega} \wedge w \leq \xi\right\} .
$$

The first result concerns the closure operator $\mathrm{cl}_{\leq}^{(\omega)}$ of $\tau_{\leq}^{(\omega)}$.

Theorem 45. Let $\leq$ be an extended partial order on $X^{\infty}$. Then $\mathrm{cl}_{\leq}^{(\omega)} F=\operatorname{Adh}_{\leq} \operatorname{Pref} F$ is the closure of $F \subseteq X^{\omega}$ in the topology $\tau_{\leq}^{(\omega)}$.

Proof. Since $\tau_{\leq}^{(\omega)}$ is the topology on $X^{\omega}$ induced by $\tau_{\leq}$, we have $\mathrm{cl}_{\leq}^{(\omega)}(F)=\mathrm{cl}_{\leq}(F) \cap X^{\omega}$. Now the assertion follows from Corollary 43.

In connection with Lemma 44 this result establishes conditions for the limit of an increasing family of words $w_{0}<\cdots<$ $w_{j}<w_{j+1}<\cdots$ to have a unique limit point in $X^{\omega}$. A necessary condition for this is obviously, that the topology $\tau_{\leq}^{(\omega)}$ should have the singletons $\{\xi\}, \xi \in X^{\omega}$, as closed sets. We now investigate this issue for the partial orders of Example 21 . 


\subsection{Quasi-lexicographical and lexicographical order}

The case of the quasi-lexicographical order $\leq_{\text {q-lex }}$ is trivial.

Example 46. The topology on $X^{\omega}$ induced by $\tau_{\leq_{\mathrm{q}-\text { lex }}}$ is trivial: only $\emptyset$ and $X^{\omega}$ are open, as $w \leq_{\mathrm{q}-\mathrm{lex}} \xi$ for all $w \in X^{*}$ and $\xi \in X^{\omega}$.

For the case of the lexicographical order some preliminary considerations are needed. Regard the alphabet $X$ as the set of non-zero $q$-ary digits $X=\{1, \ldots, q-1\}$ where card $X=q-1$, and identify an $\infty$-word $\eta \in X^{\infty}$ with the $q$-ary expansion $0 . \eta$ of a number in the real interval $[0,1]$. For $\omega$-words this yields an injective and continuous mapping $v$ from $X^{\omega}$ into the interval $[0,1]$ the image of which, $v\left(X^{\omega}\right)$, is closed.

Example 47. For $w \in X^{*}$ and $\xi \in X^{\omega}, w \leq_{\text {lex }} \xi$ if and only if $v(w) \leq v(\xi)$. This implies that, for $\zeta, \xi \in X^{\omega}, v(\zeta) \leq v(\xi)$ if and only if $\operatorname{Pred}_{\leq_{\text {lex }}} \zeta \subseteq \operatorname{Pred}_{\leq \text {lex }} \xi$. Thus the topology on $X^{\omega}$ induced by $\tau_{\leq \text {lex }}$ is homeomorphic to the right topology on the closed subset $v\left(X^{\omega}\right)$ of the unit interval. Among its closed sets, only the set $\left\{1^{\omega}\right\}$ is finite. All other closed sets are infinite. Note that $v\left(1^{\omega}\right)$ is the minimum of $v\left(X^{\omega}\right)$.

\subsection{Subword topology and disjunctive $\omega$-words}

The topology $\tau_{\leq j}^{(\omega)}$, also known as the subword topology, was investigated in [16,60]. To study it, the following notion of disjunctivity is useful.

Definition 48 ([29]). An $\omega$-word $\xi \in X^{\omega}$ is disjunctive if $w \leq_{\mathrm{i}} \xi$ for all $w \in X^{*}$.

The subword topology on $X^{\omega}$ has the following property.

Example 49 ([60]). The topology on $X^{\omega}$ induced by $\tau_{\leq_{i}}$ has the set of all disjunctive $\omega$-words as the intersection of all its non-empty open sets, that is, the closure of every singleton set $\{\xi\}$, where $\xi$ is disjunctive, is the whole space $X^{\omega}$. The only closed singleton sets in this topology are the sets $\left\{a^{\omega}\right\}$ where $a \in X$.

\subsection{Embedding order}

The investigation of the topology $\tau_{\leq \mathrm{e}}^{(\omega)}$ induced by the embedding order can be carried out in a manner analogous to the subword topology (see also [16]). Here the $\omega$-words containing each letter $a \in X$ infinitely often play the same rôle as the disjunctive words in the case of the subword topology.

Example 50. The topology on $X^{\omega}$ induced by $\tau_{\leq \mathrm{e}}$ has the set of all $\omega$-words containing each letter $a \in X$ infinitely often as the intersection of all its non-empty open sets, that is, the closure of every singleton $\{\xi\}$, where $\xi$ contains each letter infinitely often, is the whole space $X^{\omega}$. The only closed singletons in this topology are the sets $\left\{a^{\omega}\right\}$ where $a \in X$.

\section{Final comments}

We have identified some principles of inference by which sequences of finite words are extrapolated to infinite words and by which continuous functions on words can be defined. These principles are not restricted to the prefix order of words itself, but still rely on it quite heavily. It should be possible to derive far more general principles which apply to many more relations between words by changing the intuition about words being read left to right. Our main point in this paper is to focus on the underlying topologies and to expose the difficulty of defining meaningful topologies on $X^{\infty}$.

\section{Acknowledgement}

This research was supported in part by the Natural Sciences and Engineering Research Council of Canada.

\section{References}

[1] T. Ang, J.A. Brzozowski, Continuous languages. In Csuhaj-Varjú and Ésik [14] 74-85.

[2] A. Apostolico, String editing and longest common subsequences. In Rozenberg and Salomaa [52] 2, 361-398.

[3] J. Bertrema, Topologies sur des espaces ordonnés, RAIRO Inform. Théor. 16 (1982) 165-182

[4] L. Boasson, M. Nivat, Adherences of languages, J. Comput. System Sci. 20 (1980) 285-309.

[5] C.S. Calude, H. Jürgensen, L. Staiger, Topology on strings, in: Joint Workshop: Domains VIII and Computability over Continuous Data Types, Novosibirsk, 11-15 September, 2007.

[6] C.S. Calude, H. Jürgensen, M. Zimand, Is independence an exception? Appl. Math. Comput. 66 (1994) 63-76.

[7] C.S. Calude, Sur une classe de distances dans un demi-groupe libre, Bull. Math. Soc. Sci. Math. R. S. Roumanie (N.S.) 17 (65) (1973) $123-133$.

[8] C.S. Calude, On the metrizability of a free monoid, Discrete Math. 15 (1976) 307-310.

[9] C.S. Calude, V.E. Căzănescu, On topologies generated by Moisil resemblance relations, Discrete Math. 25 (1979) $109-115$.

[10] C.S. Calude, S. Marcus, L. Staiger, A topological characterization of random sequences, Inform. Process. Lett. 88 (2003) $245-250$.

[11] C.S. Calude, K. Salomaa, S. Yu, Additive distance and quasi-distances between words, J. UCS 8 (2002) 141-152.

[12] Y.A. Choueka, Structure automata, IEEE Trans. Comput. C-23 (1974) 1218-1227. 
[13] A.H. Clifford, G.B. Preston, The Algebraic Theory of Semigroups, Vols. I, II, in: Mathematical Surveys, vol. 7, American Mathematical Society, Providence, RI, 1961, 1967.

[14] E. Csuhaj-Varjú, Z. Ésik (Eds.), Automata and Formal Languages. 12th International Conference, AFL 2008, Balatonfüred, Hungary, 27-30 May, 2008. Proceedings, Computer and Automation Research Institute, Hungarian Academy of Sciences, 2008.

[15] K. Culik II, A. Salomaa, On infinite words obtained by iterating morphisms, Theoret. Comput. Sci. 19 (1982) 29-38.

[16] V.R. Dare, R. Siromoney, Subword topology, Theoret. Comput. Sci. 47 (1986) 159-168.

[17] A. Dincă, Sur quelques problèmes d'analyse contextuelle métrique, Rev. Roumaine Math. Pures Appl. 13 (1968) 65-70.

[18] A. Dincă, Distanţe contextuale în lingvistica algebrică, Stud. Cerc. Mat. 25 (1973) 223-265.

[19] A. Dincă, Distanţe şi diametre într-un semigrup (cu aplicaţii la teoria limbajelor), Stud. Cerc. Mat. 25 (1973) 359-378.

[20] A. Dincă, The metric properties on the semigroups and the languages, in: A. Mazurkiewicz (Ed.), Mathematical Foundations of Computer Science 1976 Proceedings, 5th Symposium, Gdánsk, 6-10 September, 1976, in: Lecture Notes in Computer Science, vol. 45, Springer-Verlag, Berlin, 1976, pp. 260-264.

[21] R. Engelking, General Topology, PWN, Warszawa, 1977.

[22] M. Hall Jr., A topology for free groups and related groups, Ann. of Math. 52 (1950) 127-139.

[23] T. Head, Adherence equivalence is decidable for D0L languages, in: M. Fontet, K. Mehlhorn (Eds.), STACS 84: Symposium of Theoretical Aspects of Computer Science, Paris, 11-13 April, 1984, in: Lecture Notes in Computer Science, vol. 166, Springer-Verlag, Berlin, 1984, pp. $241-249$.

[24] T. Head, The adherences of languages as topological spaces, in: M. Nivat, D. Perrin (Eds.), Automata on infinite words. Ecole de printemps d'informatique théorique, Le Mont Dore, 14-18 May, 1984, in: Lecture Notes in Computer Science, vol. 192, Springer-Verlag, Berlin, 1985, pp. $147-163$.

[25] T. Head, The topological structure of adherences of regular languages, RAIRO Inform. Théor. Appl. 20 (1986) 31-41.

[26] T. Head, Adherences of DOL languages, Theoret. Comput. Sci. 31 (1984) 139-149.

[27] P.-C. Héam, Automata for pro-V topologies, in: S. Yu, A. Păun (Eds.), Implementation and Application of Automata, 5th International Conference, CIAA 2000, London, Ontario, Canada, July 2000, Revised Papers, in: Lecture Notes in Computer Science, vol. 2088, Springer-Verlag, Berlin, 2001, pp. 135-144.

[28] S. Istrail, Some remarks on non-algebraic adherences, Theoret. Comput. Sci. 21 (1982) 341-349.

[29] H. Jürgensen, H.J. Shyr, G. Thierrin, Disjunctive $\omega$-languages, Elektron. Informationsverarbeit. Kybernetik. 19(6) (1983) $267-278$.

[30] H. Jürgensen, L. Kari, G. Thierrin, Morphisms preserving densities, Internat. J. Comput. Math. 78 (2001) 165-189.

[31] H. Jürgensen, S. Konstantinidis, Codes. in Rozenberg and Salomaa [52] 1, 511-607.

[32] H. Jürgensen, I. McQuillan, Homomorphisms preserving types of densities. in Csuhaj-Varjú and Ésik [14] 183-194.

[33] H. Jürgensen, G. Timmermann, Unscharfe Dominanz und ihre Berechnung, Rev. Roumaine Math. Pures Appl. 25 (1980) 871-878.

[34] W. Kuich, Semirings and formal power series: Their relevance to formal languages and automata. in Rozenberg and Salomaa [52] 1, 609-677.

[35] K. Kuratowski, Topology I, Academic Press, New York, 1966.

[36] M. Latteux, E. Timmermann, Two characterizations of rational adherences, Theoret. Comput. Sci. 46 (1986) $101-106$.

[37] V.I. Levenshtein, Двоичные коды ц исправлением выпадений, вставок и замещений символов, Dokl. Akad. Nauk. SSSR 163 (4) (1965) 845-848. English translation: Binary codes capable of correcting deletions, insertions, and reversals, Sov. Phys. Dokl. 10 (1966) $707-710$.

[38] R. Lindner, L. Staiger, Algebraische Codierungstheorie; Theorie der sequentiellen Codierungen, Akademie-Verlag, Berlin, 1977.

[39] M. Malitza, Topology, binary relations and internal operations, Rev. Roumaine Math. Pures Appl. 22 (1977) 515-519.

[40] S. Marcus, Introduction mathématique à la linguistique structurale, Dunod, Paris, 1966.

[41] E. Nelson, Categorical and topological aspects of formal languages, Math. Systems Theory 13 (1980) 255-273.

[42] M. Nivat, Infinite words, infinite trees, infinite computations, in: J.W. de Bakker, J. van Leeuwen (Eds.), Foundations of Computer Science III Part2: Languages, Logic, Semantics, in: Mathematical Centre Tracts, vol. 109, Mathematisch Centrum, Amsterdam, 1979, pp. 3-52.

[43] D. Perrin, J.-É. Pin, Infinite Words, Elsevier, Amsterdam, 2004.

[44] J.E. Pin, Topologies for the free monoid, J. Algebra 137 (1991) 297-337.

[45] J.-E. Pin, Polynomial closure of group languages and open sets of the Hall topology, in: S. Abiteboul, E. Shamir (Eds.), Automata, Languages and Programming, 21st Internat. Coll., ICALP94, Jerusalem, Israel, July1994, Proceedings, in: Lecture Notes in Computer Science, vol. 820, Springer-Verlag, Berlin, 1994, pp. 424-435.

[46] J.-E. Pin, C. Reutenauer, A conjecture on the Hall topology for the free group, Bull. London Math. Soc. 23 (1991) 356-362.

[47] H. Prodinger, Topologies on free monoids induced by closure operators of a special type, RAIRO Inform. Théor. 14 (1980) $225-237$.

[48] H. Prodinger, Topologies on free monoids induced by families of languages, RAIRO Inform. Théor. 17 (1983) 285-290.

[49] V.T. Raischi, O distanță definită în limbajele semantice, Stud. Cerc. Mat. 26 (1974) 265-279.

[50] R.R. Redziejowski, Infinite word languages and continuous mappings, Theoret. Comput. Sci. 43 (1986) 59-79.

[51] T. Richter, Lokalisierung in formalen Sprachen, Diplomarbeit, Institut für Informatik, Martin-Luther-Universität Halle-Wittenberg, 2007.

[52] G. Rozenberg, A. Salomaa (Eds.), Handbook of Formal Languages, Springer-Verlag, Berlin, 1997.

[53] W. Shukla, A.K. Srivastava, A topology for automata: A note, Inform. Control 32 (1976) 163-168.

[54] H.J. Shyr, Free Monoids and Languages, third ed., Hon Min Book Company, Taichung, 2001, iv+282 pp.

[55] R. Siromoney, V.R. Dare, On infinite words obtained by selective substitution grammars, Theoret. Comput. Sci. 39 (1985) $281-295$.

[56] M.B. Smyth, Topology, in: S. Abramsky, D.M. Gabbay, T.S.E. Maibaum (Eds.), Handbook of Logic in Computer Science, vol. 1, Clarendon Press, Oxford, 1992, pp. 641-761.

[57] L. Staiger, Über ein Analogon des Satzes von Ginsburg-Rose für sequentielle Folgenoperatoren und reguläre Folgenmengen. Diplomarbeit, Sektion Mathematik, Friedrich-Schiller-Universität Jena, 1970

[58] L. Staiger, Sequential mappings of $\omega$-languages, RAIRO Inform. Théor. 21 (1987) 147-173.

[59] L. Staiger, $\omega$-languages. in Rozenberg and Salomaa [52] 3, 339-387.

[60] L. Staiger, Topologies for the set of disjunctive $\omega$-words, Acta Cybernet. 17 (2005) 43-51.

[61] L. Staiger, K. Wagner, Automatentheoretische und automatenfreie Charakterisierungen topologischer Klassen regulärer Folgenmengen, Elektron. Informationsverarbeit. Kybernetik. 10 (1974) 379-392.

[62] W. Thomas, Automata on infinite objects, in: J. van Leeuwen (Ed.), Handbook of Theoretical Computer Science B, North-Holland, Amsterdam, 1990, pp. 135-191.

[63] R. Valk, Topologische Wortmengen, topologische Automaten, zustandsendliche stetige Abbildungen, Mitteilungen der Gesellschaft für Mathematik und Datenverarbeitung, vol. 19, GMD, Bonn, 1972.

[64] H. Walter, Topologies on formal languages, Math. Systems Theory 9 (1975) 142-158.

[65] S.-S. Yu, Languages and Codes, Tsang Hai Book Publishing Co., Taichung, Taiwan, 2005.

[66] B. Zelinka, Un langage avec la distance contextuelle finie, mais non bornée, Sborník Védeckých Prací Vysoké Školy Strojní a Textilní v Liberci 6 (1967) 9-12. 